\title{
A Novel Delay- and Reliability- Aware Inter-Vehicle Routing Protocol
}

\author{
Kayhan Zrar Ghafoor, Kamalrulnizam Abu Bakar, and Haidar N. AL-Hashimi \\ Faculty of Computer Science \& Information Systems \\ Universiti Teknologi Malaysia, 81310 UTM Skudai, Johor D. T, Malaysia
}

Tel: 006-012-7792943 E-mail:zgkayhan2@live.utm.my

\author{
Kevin C. Lee \\ Department of Computer Science, \\ University of California, Los Angeles, CA 90095, USA
}

Received: June 27, 2010 Accepted: July 14, 2010 DOI: 10.5296/npa.v2i2.427

\begin{abstract}
Intelligent transportation systems could improve transportation safety, driving assistance and traffic management system. Vehicular Ad hoc Network (VANET) is an emerging field of technology, embedding wireless communication networks into vehicles to achieve intelligent transportation systems. The development of such systems pose many unique challenges like designing routing protocols that not only forward packets with good end to end delay but also take into consideration the reliability and progress in data packets forwarding. In this article, we begin by presenting a review of recent unicast, geocast and broadcast routing protocols for message transmission. We then outline a novel Delay and Reliability aware Routing (DR $\left.{ }^{2}\right)$ protocol that addresses these challenges (forwarding packets with low latency, high reliability and fast progress toward destination). Furthermore, our $\mathrm{DR}^{2}$ protocol uses cross layer communication between MAC (Medium Access Control) and network layer. That is, the MAC layer observes the Signal to Noise (SNR), delay and velocity vector difference metrics for all paths of neighboring nodes, network layer then could select the best preferable path based on fuzzy inference system. We also used $\mathrm{H}_{\infty}$ technique to optimize the membership functions and then tune it with rapid changing topology of VANET. To achieve a fair comparison with other routing protocols, we have implemented the proposed $\mathrm{DR}^{2}$ protocol in Network Simulator 2 (NS 2). The preliminary results show that the proposed $\mathrm{DR}^{2}$ protocol is able to improve end- to-end delay in sparse traffic conditions and packet delivery ratio in error prone urban vehicular scenarios.
\end{abstract}


Keywords: VANET, Fuzzy Inference System, Hœ technique, Cross Layer Communication, Routing Protocol.

\section{Introduction}

The continuous increase in the number of vehicles along the transportation system has made driving more challenging and unsafe. In this perspective, the current transportation systems need an improvement of traffic safety, efficiency and overall traffic infrastructure. To achieve this demand, the Intelligent Transportation Systems (ITS) have been considered to enable various road traffic applications such as traffic safety, cooperative traffic monitoring and control of traffic flow. VANET is a significant component of ITS, through which many ITS applications can become realities. Vehicular wireless communication networks not only could provide scalable connectivity between vehicles on the road, but also can establish better coordination between road mobile users. Preeminent car manufactures around the globe have been jointly working with international research communities to integrate wireless communication devices in their production. This technology is beneficial to help drivers anticipate risky events and avoid traffic jam. In the near future the number of vehicles equipped with communication device are going to increase, thus the scalable VANET can be envisioned in the near future. A number of research projects around the world, e.g. [1] in Europe, [2] in US and [3] in Japan are engaged in researching and developing the infrastructure of VANET and ITS.

Recently, the main objective of VANET has clearly been to enhance traffic safety of transportation system. In addition to that, car manufactures and academia community have been researching new VANET applications such as on road entertainment and traffic safety management. On the other hand, VANET applications are necessities more exploring in multi-hop routing between vehicles. This application enables large area coverage between vehicles at the same time which is departure from traffic emergency applications that require short range communication.

However, designing a multi-hop routing protocol to well aware end to end delay and the quality of wireless channel between vehicles are daunting task. The reasons of this are high mobility, frequent topology fragmentation and frequent disconnection between vehicles. Furthermore, the communication channel between vehicles is prone to radio frequency interference and shadowing together with different forms of fading including multi-path and slow fading. As a consequence, the channel suffers both from high bit error rate and high packet error rate which leads to high packet loss [4]. Under this condition, existing protocols designed for Mobile Ad hoc Networks (MANET) [15] can not directly applied on VANET. The majority of current techniques developed for VANET are position based rather than address based. The position based routing is desirable in VANET for the following reasons. First, it's beaconing easier to support position based routing as Global Positioning System (GPS) are becoming cheaper. Second, it does not maintain established routes. Third, the designed routing protocols suffer from high overhead.

The recent multi-hop position based routing protocols do not take into consideration the lossy wireless channel due to multi-path fading and shadowing. Moreover, some of the 
position based routing protocols select farthest forwarding node from the source to retransmit the message to the required destination [19]. The problem of this forwarding approach is that as the distance between source and rebroadcasting node increases the severity of signal attenuation is also increases. The reason of this, higher distance leads to higher path loss, then yields higher attenuation. As a consequence, the network packet delivery ratio degrades and routing packet overhead significantly increases. Furthermore, the previous solutions do not take delay into account. Thus, they fail to transmit packets reliably over minimum delay paths.

In this article, we review the broadcast, geocast, and unicast routing approaches for transmitting data message. We then propose a $\mathrm{DR}^{2}$ protocol that addresses the above mentioned challenges (see detail in Section 5). The proposed protocol relies on the cross layer communication between MAC and network layers, in such away MAC layer observes and update path metrics like delay, SNR and velocity vector difference of the neighboring nodes. After that fuzzy inference system, embedded in the network layer, selects a route based on the observed metrics. The proposed protocol achieves lower end to end delay in sparse traffic condition and robustness under lossy wireless channel. It is worth to mention that the proposed routing protocol is distributed in such away source and relay nodes make high level of route decisions.

The rest of the paper is organized as follow: Section 2 describes the Inter-Vehicle Communication (IVC) routing protocols for transmitting data message. The proposed $\mathrm{DR}^{2}$ is discussed in section 3.Finally, section 4 concludes the paper.

\section{IVC Protocols for Message Transmission}

Taking into consideration all the above mentioned unique characteristics, a different type of routing method is required in order to cope with the VANET environment. Each of the existing protocols found in the literature has different architectural design, regarding to the data link, network, and transport layers of OSI model. Hence, a good design decision has a positive effect on multidimensional aspects of VANET communication services. Also there is a trade-off between metrics for instance, increasing delay for higher throughput which need to be tackled during the designing process. Fig. 1 shows a tree structure of classified IVC protocols which are used for unicasting, geocasting and broadcasting information. In the following sections the types of IVC protocols are discussed.

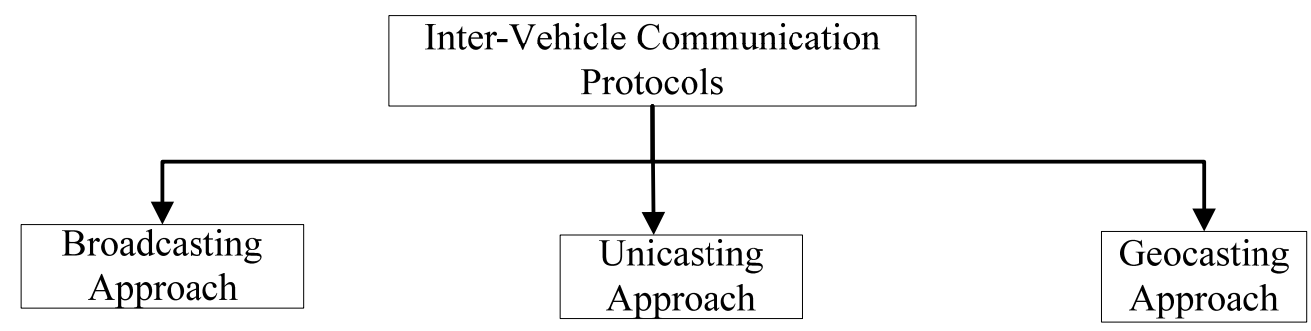

Figure 1. Taxonomy of Inter-Vehicle Communication protocols. 


\subsection{Broadcast Protocols}

The objective of broadcasting in VANET is to disseminate information from one source to many destinations. In the other words, broadcasting is a method of information dissemination which is frequently used in VANET. Some of possible applications relying on broadcast include emergency data dissemination, road traffic data sharing among vehicles, and delivering advertisement and announcement [5]. The broadcasting in the mobile wireless networks of (n) nodes costs (n) transmissions. Therefore, it offers more overhead and bandwidth utilization for improved reliability. Some authors [6] have proposed the method for restricting the rebroadcasting nodes while maintaining radio coverage. The Urban Multi-hop Broadcast protocol (UMB) in [5] introduces a new request-to-send and clear-to-send handshake for IEEE 802.11 that enables the farthest node from a transmitter to retransmit packets. However, the potential relay nodes wait for the longest time before retransmitting. This mechanism may lead to long latency, especially for high node densities. Some authors [7] have proposed a solution of UMB by designing a position-aware routing protocol, named Smart Broadcast, for fast and reliable message propagation in VANET. It makes use of position information provided by a GPS-like system to speed up the message propagation along the network, by allowing farthest nodes to attempt transmission first.

In addition, the high velocity and limited communication range of the vehicles incur frequent link disconnection and even network partition. It takes time for vehicle to catch up with other vehicles ahead and reconnect the network. This amount of time is called as catch-up delay [8]. During the catch-up phase, the routing protocol uses the store-and-forward scheme to buffer the packet and send it in the next chance [9]. Some authors in [10] have designed Directional Propagation Protocol (DPP) for data dissemination in VANET using opportunistic forwarding. Fig. 2 illustrates the formation of clusters and how Directional Propagation Protocol (DPP) can provide communication between vehicles whenever clusters are fully connected. Thus, in VANET, the opportunistic flooding is used for packet dissemination over intermittent connectivity. This type of packet dissemination is often leads to a higher proportion of delivered messages and lowers transmissions, but message propagation may be relatively slow. A vehicle may use GPS to decide whether a rebroadcast will advance stored messages following a period of motion. Authors in [11] have presented a broadcast routing protocol. This broadcast approach adapts with different vehicular traffic conditions. The protocol could achieve less number of transmissions while maximizing reliability. 


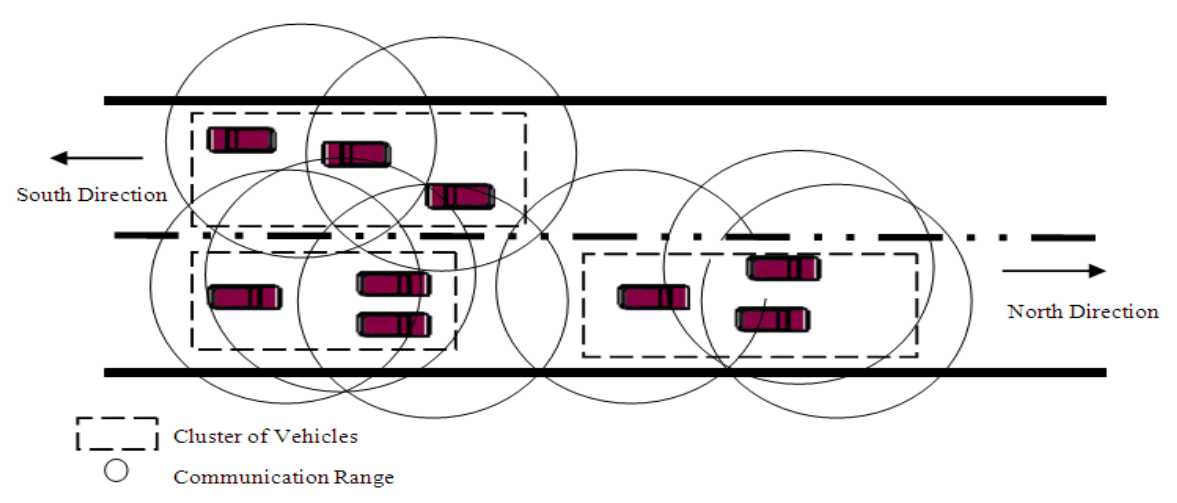

Figure 2. Illustration of multi-hop routing between clusters.

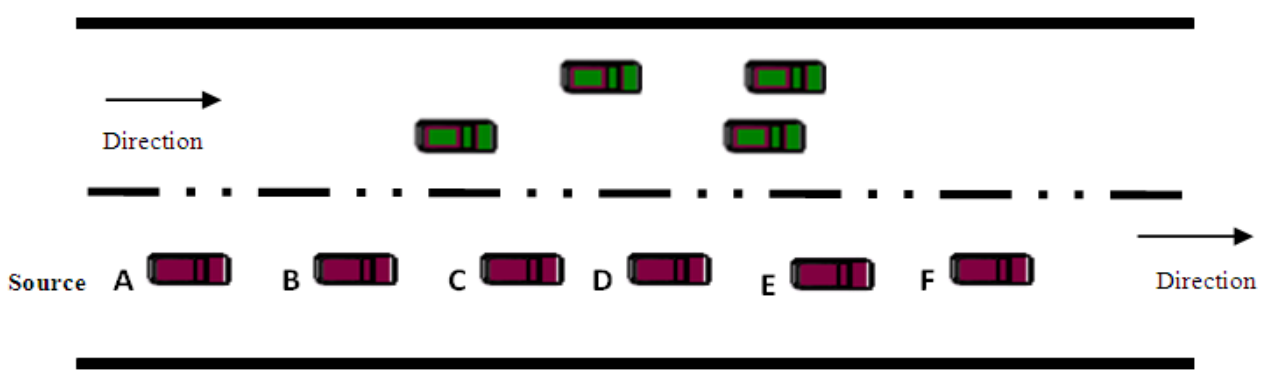

Figure 3 Common vehicular scenario.

The given scenario illustrated in Fig. 3. vehicle A is a source of broadcast message which is received by $\mathrm{B}, \mathrm{C}$, and $\mathrm{D}$. After that receivers set up a defer time which is shorter if vehicles belongs to the computed Connected Dominating Set (CDS). Let D be in the CDS, so it retransmit first. As a result $\mathrm{D}$ covers all neighbors thus $\mathrm{B}$ and $\mathrm{C}$ cancel their transmission. Then all covered intended receivers acknowledge the reception of the message.

The lack of acknowledgment means the receivers are not fully covered. Some authors [12] have proposed Reliable Broadcast based on Mobility Prediction (RB-MP) for VANET. Firstly, the protocol divides the neighbors in the base of their direction. Then the rebroadcast node is selected by using the position and velocity information.

The mobility-centric data dissemination (MDDV) protocol [13] uses opportunistic forwarding to propagate a message along a trajectory and deliver it to geographical destination region by a deadline. Opportunistic forwarding targets networks when an end-to-end path can not be assumed to exist. Messages are stored and forwarded to another node; a copy may remain with the original and be forwarded again later to improve reliability. Fig. 4 shows the concept of opportunistic forwarding in MDDV.

\subsection{Geocast Protocols}

Efficient message deliveries in VANET to a group of intend receivers is achieved by using group communication. The group of intended receivers could be more effective if it is designed as narrowly as possible. In order to maximize the performance of the protocol and 
preserve the network resources, the set of intended receivers can be identified by geographical coordinates. The objective of using geographic coordinates is to constrain the scope of massage forwarding to a set of receivers and the technique is called geocast [14].

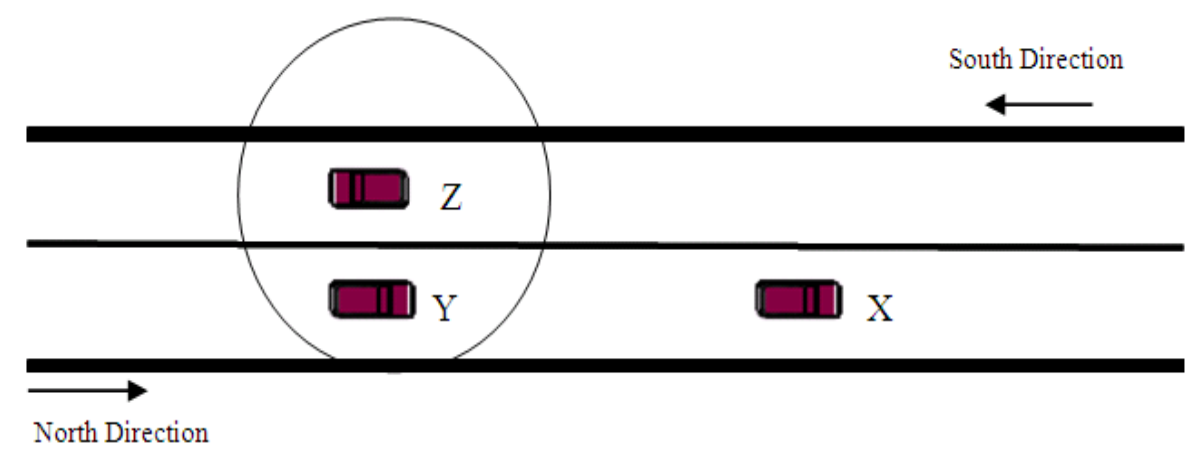

Figure 4. Opportunistic forwarding: vehicle $\mathrm{Z}$ stores transmitted message from vehicle $\mathrm{X}$ to vehicle $\mathrm{Y}$.

The node which receives an alarm message or warning should not rebroadcast it immediately but it has delay in time, called defer time, to take a decision about rebroadcast. When this defer time expires and if it does not receive the same alarm message from another node behind it, it deducts that there is no relay node behind it. Thus, it has to designate itself as a relay and broadcasting alarm messages to inform the vehicles which might be behind it. Hence, a broadcast storm is avoided and the forwarding is optimized around the initiating vehicle.

Besides, classical geocast routing, recently, abiding geocast is introduced to disseminate information to a group of nodes in an area for specific time interval as discussed in [16]. When a vehicle enters such an area, the virtual warning sign displayed for the driver it employed periodic flooding or epidemic dissemination. The authors [17] have proposed a system of abiding geocast for disseminating warning messages in VANETs. Two strategies are used to improve the efficiency of disseminating i.e. vehicles travelling in opposite direction are used as preferred relays to overcome fragmentation and reduce broadcasting. Fig. 5 shows the illustration of abiding geocast, in which the vehicle in the problem area inform all vehicles moving left before they pass the safety line during the life time of the event, at the same time vehicles broadcast as few messages as possible.

\subsection{Unicast Protocols}

Unicast protocols provide information delivery between two nodes via multiple wireless hops. Unicast communication between vehicles is more complicated due to frequent topology fragmentation and bipolar traffic conditions: sparse and dense scenarios. Most ad hoc routing protocols that discover and maintain end to end paths (e.g., Ad Hoc on Demand Vector [AODV][15]) are less preferable due to high protocol overheads. The reason of this overhead is that the path establishment between the source and the destination needs source's flooding of route discovery packet and then the destination reply it back by sending of a route reply data packet. The established route remains active as long as the source needs it. The 
path failure will be notified repeatedly to the source and will successively actuate another route discovery response step to find a new route. As consequence of high speed of vehicles, the full duplex path establishment between the source and the destination produce more time consuming and may lead to many route failures between the source and the destination.

Authors in [18] have proposed Velocity-Headed based Routing Protocol (VHRP). This method uses GPS information to predict the path disconnection and take preemptive action.

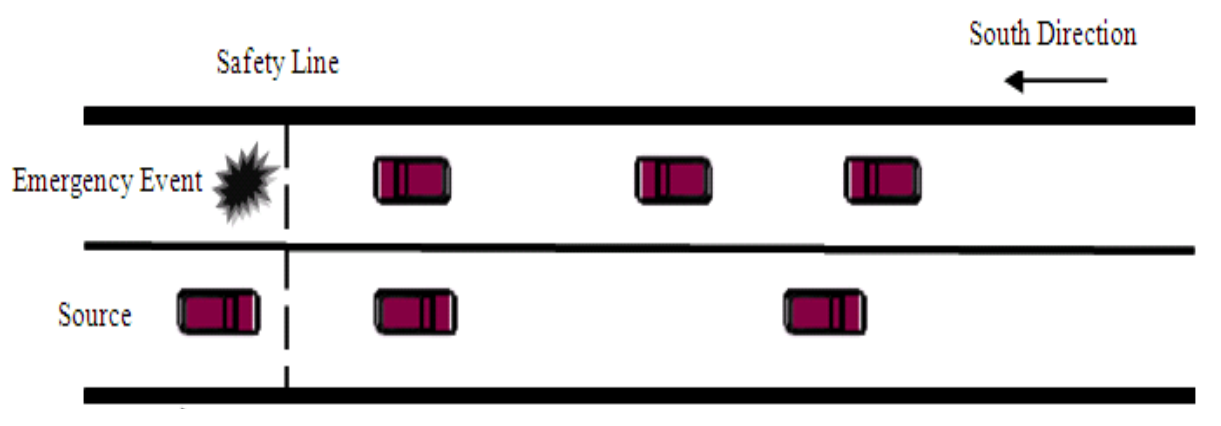

Figure 5. Emergency message dissemination.

In VANET, geographical routing has been known as more promising routing paradigm. A position based routing consists of some components such as beaconing, location service, and forwarding scheme. Hello packets are utilized to get topology information of the network whereas the location service is used to obtain the location of the receivers. Furthermore, a forwarding scheme selects the next hop based on geographical information of the current node.

The routing discovery strategy of position based forwarding algorithms based on location information. For instance, Greedy Perimeter Stateless Routing (GPSR) selects the node that is geographically closest to the packet's destination [19]. In addition to this, it combines greedy routing with perimeter backtracking. The perimeter backtracking is used to get out of the local optimum where greedy fails. However, this type of forwarding increases the packet loss due to high attenuation of the destination path.

Also the graph which is used in GPSR position based routing must be planar. Thus, some authors in [20] proposed Greedy Perimeter Coordinator Routing (GPCR). It utilizes the concept of junction nodes to control the next road segments that packet should follow. However, in mobile urban environment, the concept of junction nodes is an issue and hard to maintain. Some authors in [21] have proposed GpsrJ+. The enhancement is based a prediction method to select a road segment on which its neighboring junction node will forward packet to. In addition, to address the issue of high mobile and route breakage, authors in [22] have proposed Vehicle Assisted Data Delivery (VADD). It is adopted the idea of carry and forward approach with vehicle mobility prediction. Furthermore, some authors in [23] have proposed a distributed protocol for multi-hop data dissemination. The protocol achieves maximum packet delivery under a wide range of network conditions in vehicular networks. However, it fails to forward packets over minimum delay route between the source and the destination. Therefore, based on the above literature review, the novel routing protocol is 
indigence to smartly establish a route with minimum delay and high reliability between the source and the destination. In the next section the novel reliability and delay aware routing protocol are discussed in the following sections.

\section{Delay and Reliability aware Routing $\left(\mathrm{DR}^{2}\right)$ Design}

The $\mathrm{DR}^{2}$ is a reliable and delay aware routing protocol, designed to transmit information from the source to the destination vehicle through multi-hop communication. Our protocol is designed based on cross layer communication between MAC and network layers. The MAC layer is in charge of gathering routing metrics by tracking channel condition and calculating relative velocity. These statistics are then forwarded to the network layer to select the best neighboring vehicle through fuzzy inference system. In addition to this, the membership functions of fuzzy variables are optimized by triggering $\mathrm{H} \infty$ technique. It is worth to note that this cross layer communication has less overhead due to passing light information from the MAC to the network layer. The architecture of the proposed $\mathrm{DR}^{2}$ is illustrated in Fig. 6. All vehicles are embedded with fuzzy inference system and $\mathrm{H} \infty$ technique. The gray blocks are the scope of our $\mathrm{DR}^{2}$ protocol.
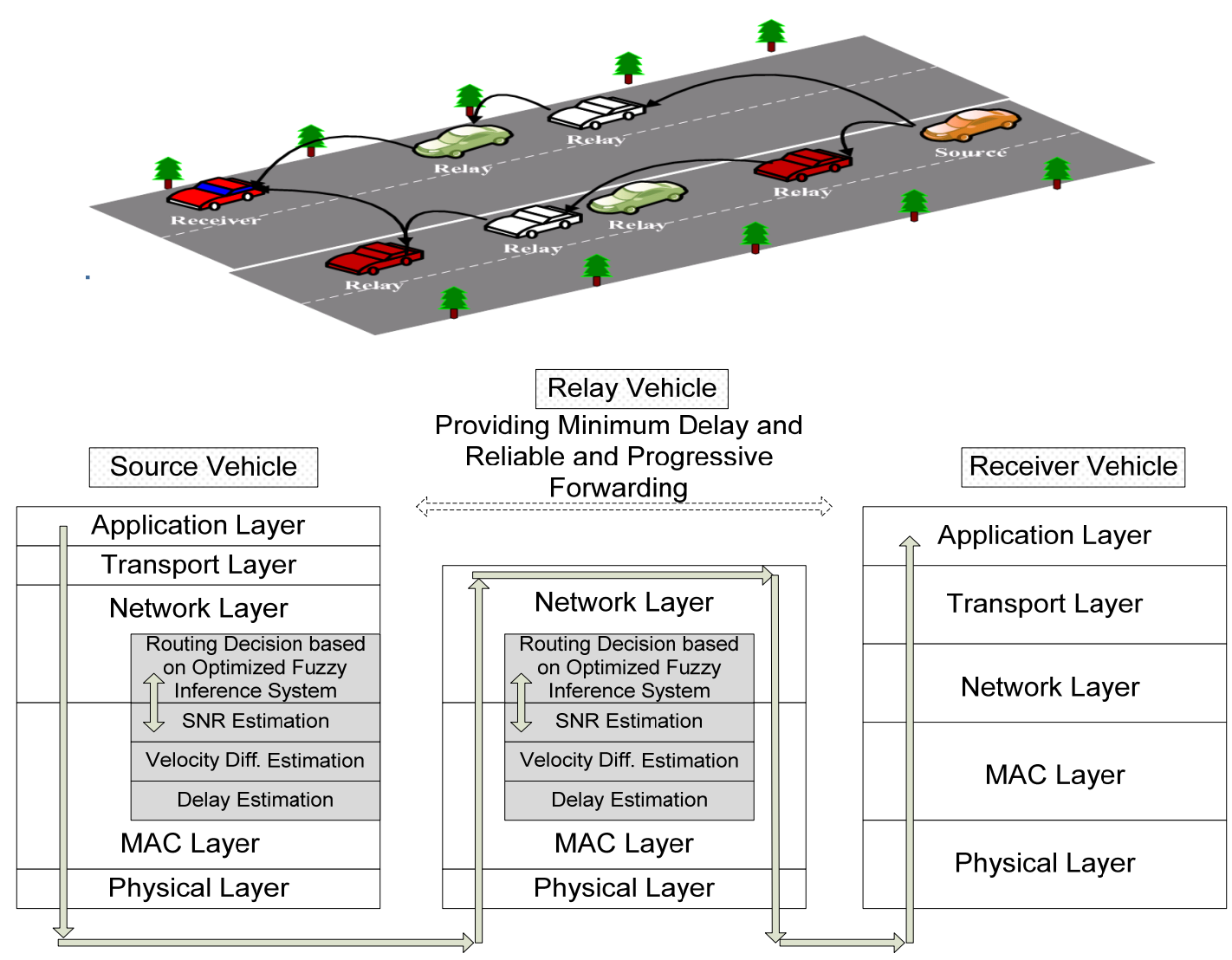

Figure 6. Overall block diagram of the proposed $\mathrm{DR}^{2}$ Protocol (the gray blocks are the scope of the proposed $\mathrm{DR}^{2}$ ). 
Similar to existing works on VANET, we assume that vehicles are embedded with GPS receiver that provides vehicles current position to all neighbors. We also assume that vehicles are embedded with digital road map to provide accurate locations of streets. Vehicles are equipped with wireless radio communication devices in order to facilitate communication. These assumptions are leveraged by MAC layer in order to easily track the metrics which are used as input to the routing layer.

\subsection{MAC Layer based Routing Metrics Gathering}

VANET is a high mobile wireless network system. For this reason, periodic update of traffic information between vehicles is necessary to provide a sufficient picture of the network condition. In this way, all vehicles could adopt to variations in the underlying vehicular environment that are located in the vicinity zone.

The $\mathrm{DR}^{2}$ protocol adopted here selects the best routing path based on three metrics (as it is mentioned in section 3). The procedural steps involved in tracking these three metrics by MAC layer are as follows:

1) SNR: Wireless channel quality between two mobile nodes can be represented by SNR. In VANET, it can be used to estimate the behavior of time varying vehicular wireless channels. We modified the Round Trip Time (RTT) approach [24] for the sake of estimating the SNR of channels of neighbor vehicles. Originally RTT approach is used to measure the delay of the wireless channel. Whereas, we modified the returned Acknowledgement frame (ACK) by including the SNR field in it. The SNR value is required of an additional one byte to store its value in $\mathrm{dB}$. In this way, when the source/relay MAC layer tracks the SNR of the channel, it sends a hello packet to all covered neighbors. After receiving the hello packet, receivers put the measured SNR in the ACK frame then send it back to the source. After that, the source can use SNR of the corresponding ACK frame.

2) Delay: similar to the SNR sensing approach, we use RTT to estimate the channel delay for each neighboring vehicle. RTT is measured by sending a hello packet to all neighbors. The receivers send ACK back to the source and then RTT is the time required for the hello packet to traverse the link between source and receivers.

3) Velocity vector Difference: Vehicles transmit periodic beacons. The beacon is broadcasted (MAC broadcast: which are used for local topology sensing only, it is not re-broadcasted) periodically and contains vehicles information such as: address (ID), location, velocity vector. Each vehicle maintains a topology table to store information of vehicles within its transmission range. Upon receiving a beacon, each vehicle updates its table with new vehicles information. The table is checked periodically by the vehicle to update (add/delete) it. Therefore, the source and relay vehicles can easily find the velocity difference for their neighbors, as demonstrated in Fig. 7, there are three relay vehicles in the radio range of the source vehicle; the source vehicle selects (this decision is made in routing layer) the vehicle with highest speed $(100 \mathrm{~km} / \mathrm{hr})$. This is because higher the velocity of the relay vehicle, the greater 
the progress of the data packet to reach the destination. When the MAC layer senses the three required routing metrics, the fuzzy inference system in the network layer selects the best relay vehicle based on its channel condition as it is demonstrated in the following sections.

\subsection{Fuzzy \& Hळ Filtering based Routing}

The routing function in network layer of the proposed $\mathrm{DR}^{2}$ protocol has two techniques: fuzzy inference system and $\mathrm{H}$-infinity filtering. The fuzzy inference system reads all fetched metrics from the MAC layer and then decides the best preferable path whereas the membership functions of the fuzzy variables has been tuned with VANET's rapid changing of topology using $\mathrm{H} \infty$ filtering.

\subsubsection{Design of Fuzzy Inference based Path Selection}

Fuzzy logic is a process of decision making based on input membership functions and a group of fuzzy rules like human brain which simulate the interpretation of uncertain sensory information. Here fuzzy logic is applied for selecting the best path, based on more than one metric, from a source to many receivers. In this case, the source does not know which path is the best, so fuzzy is the answer of this uncertain type of problem. This approach has been used for the best path decision making using combined path metrics.

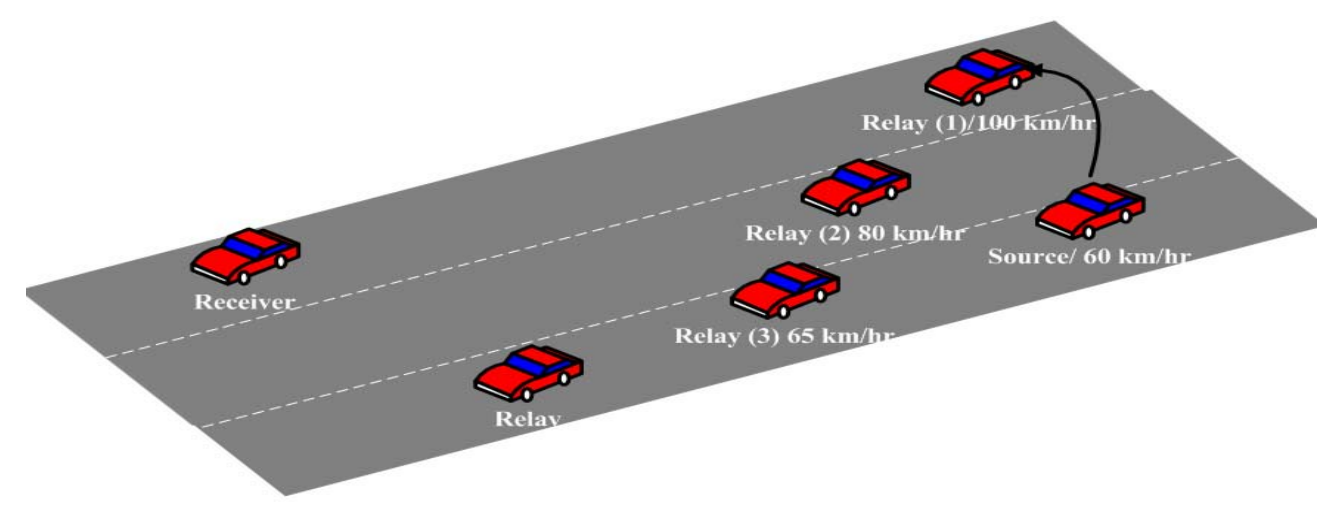

Figure 7. Relay vehicle selection based on velocity vector difference.

As demonstrated in Fig. 8, the fuzzy inference system consists of fuzzification, knowledge rule base and defuzzification. The first step of designing a fuzzy inference system is to determine membership functions to the input and output fuzzy variables based on the defined range. This is followed by designing rules for the fuzzy inference system. Furthermore, a group of rules is used to represent knowledge based for articulating the control action in linguistic form. The overall process involves in estimating the best preferable path to neighboring vehicles (in a distribute way) is elaborated as follows:

\subsubsection{Fuzzification of Inputs and Outputs}


The three input routing metrics to be fuzzified are the SNR value, the path delay and the velocity vector difference (Fig. 9). The membership functions named "Low","Medium" and "High" are used to describe the SNR. The initial selection of SNR membership functions can be derived based on experience as well as trial and error, thus the range of SNR starts from (4 $\mathrm{dB})$ and ends in $(22 \mathrm{~dB})$. The minimum value $(4 \mathrm{~dB})$ of SNR represents the high lossy channel between vehicles while it's maximum value exemplify the low error prone channel. When vehicles are in motion the value of SNR for the channel between vehicles are varied between its minimum to its maximum value. Thus, when a source/relay vehicle wants to transmit data packets, it first checks the value of SNR of the neighbor vehicles and then it selects the vehicle with high SNR.

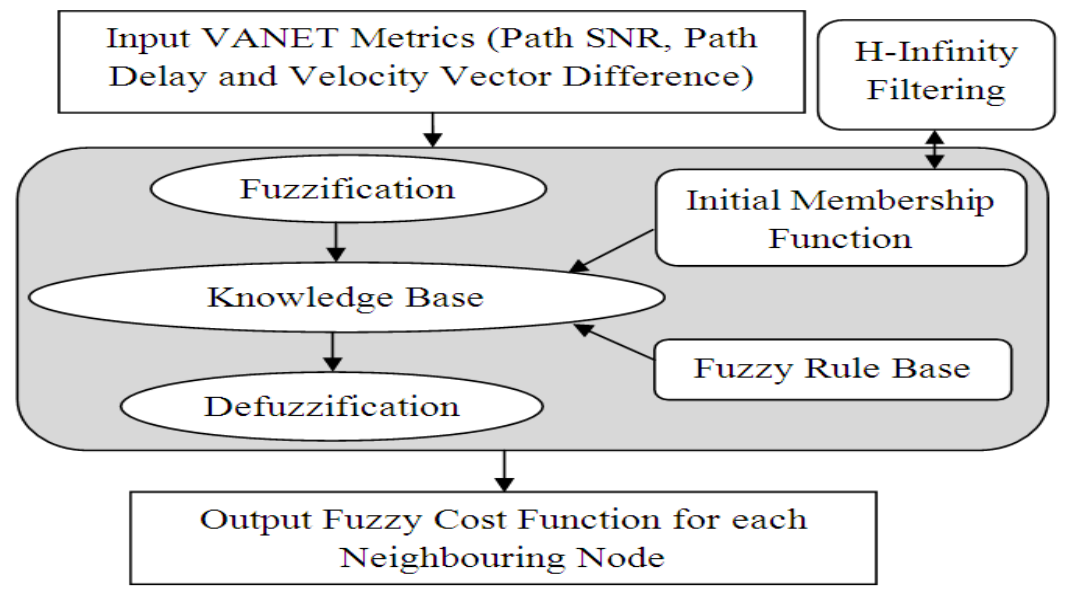

Figure 8. Computation of fuzzy cost when source and intermediate node selects a path

In the same way, the membership functions of the delay fuzzy variable are "LowD", "MediumD" and "HighD". The normalized range which represents the membership functions starts from (0.1) to (0.9) (Fig. 9). The path delay between vehicles oscillates with the variation of vehicular topology. In a like manner, the "SmallVD","MediumVD" and "LargeVD" are membership functions for representing the velocity difference. As demonstrated in section (3.4.1), vehicles on our urban simulation scenario are travelling with a maximum speed of $60 \mathrm{~km} /$ hour. Thus, based on this speed we have set the range of velocity difference variable. The range of this fuzzy variable starts from $(10 \mathrm{~km} / \mathrm{hr})$ and ends in $(50 \mathrm{~km} / \mathrm{hr})$. In other words, vehicles are travelling in different speed on multi lane urban scenario. Furthermore, the output membership functions of the fuzzy cost variable are "VLow", "Low", "Medium", "High", "VHigh" (Fig. 10). The range of the output fuzzy cost function is (0.1-0.9), the greater the value the higher the probability for selecting specified path. The triangular functions are used as the membership function because they have been extensively used in real-time applications due to their simple formulas and computational efficiency. This wise design of membership functions has positive impact on the fuzzy decision making performance. 

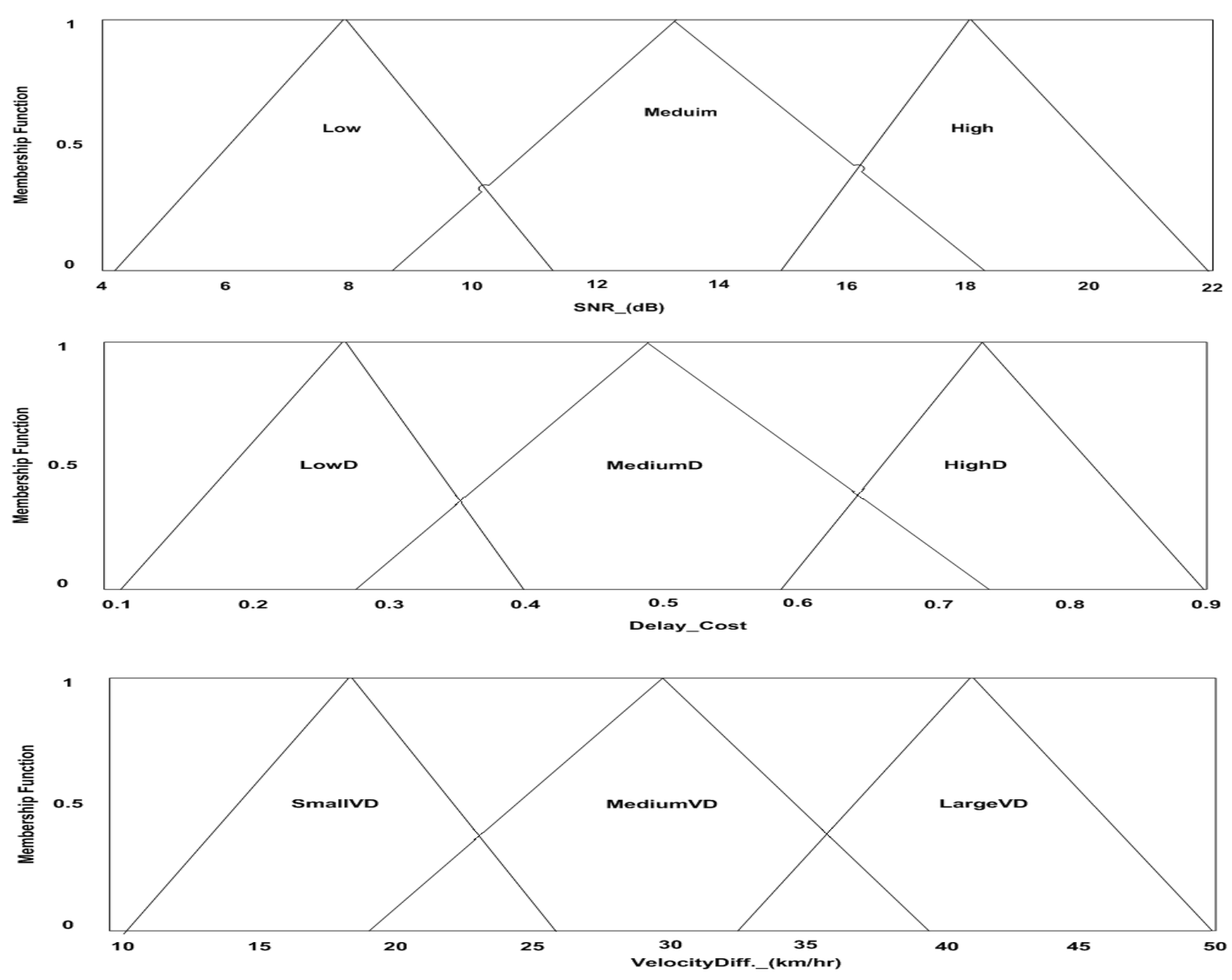

Figure 9. Fuzzy membership functions for SNR, Delay and Velocity Difference inputs

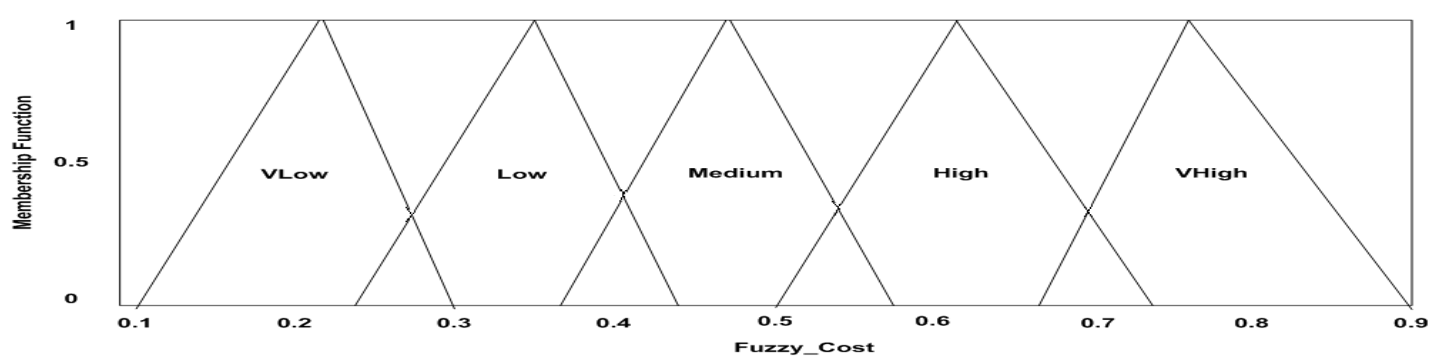

Figure 10. Fuzzy membership function for Fuzzy cost output

\subsubsection{Fuzzy Inference Engine}

The fuzzy inference engine is a group of rules developed using expert knowledge. We have designed the knowledge based rules that are connecting the inputs and the output based on a careful understanding of the philosophy behind routing metrics and vehicular networks behavior. The fuzzy inference system is designed based on 27 rules which are presented in Fig. 11. In order to demonstrate the designed fuzzy inference system, one rule is used to show how the inference engine works and the outputs of each rule are combined for generating the 
fuzzy decision [25]. Consider a rule "If SNR is High and Delay is HighD and VelocityDiff. is LargeVD, then the FuzzyCost is high" as an example of calculating output of the specified rule. In our fuzzy inference system, consider a case where SNR is $15.2 \mathrm{~dB}$ and Delay is 0.808 and VelocityDiff. is $44.9 \mathrm{~km} / \mathrm{hr}$. the output is 0.546 .

R1: IF SNR is Low \& Delay is LowD \& VelocityDiff. is SmallVD THEN Fuzzy_Cost is Low R2: IF SNR is Low \& Delay is LowD \& VelocityDiff. is MeduimVD THEN Fuzzy_Cost is Meduim R3: IF SNR is Low \& Delay is LowD \& VelocityDiff. is LargeVD THEN Fuzzy_Cost is High R4: IF SNR is Low \& Delay is MediumD \& VelocityDiff. is SmallVD THEN Fuzzy_Cost is VLow R5: IF SNR is Low \& Delay is MediumD \& VelocityDiff. is MediumVD THEN Fuzzy_Cost is Low R6: IF SNR is Low \& Delay is MediumD \& VelocityDiff. is LargeVD THEN Fuzzy_Cost is Medium R7: IF SNR is Low \& Delay is HighD \& VelocityDiff. is SmallVD THEN Fuzzy_Cost is VLow R8: IF SNR is Low \& Delay is HighD \& VelocityDiff. is MeduimVD THEN Fuzzy_Cost is Low R9: IF SNR is Low \& Delay is HighD \& VelocityDiff. is LargeVD THEN Fuzzy_Cost is Meduim R10: IF SNR is Medium \& Delay is LowD \& VelocityDiff. is SmallVD THEN Fuzzy Cost is Low R11: IF SNR is Medium \& Delay is LowD \& VelocityDiff. is MediumVD THEN Fuzzy_Cost is High R12: IF SNR is Medium \& Delay is LowD \& VelocityDiff. is LargeVD THEN Fuzzy_Cost is VHigh R13: IF SNR is Medium \& Delay is MediumD \& VelocityDiff. is SmallVD THEN Fuzzy_Cost is Low R14: IF SNR is Medium \& Delay isMediumD\&VelocityDiff. isMediumVDTHENFuzzy_Cost is Medium R15: IF SNR is Medium \& Delay is MediumD \& VelocityDiff. is LargeVD THEN Fuzzy_Cost is High R16: IF SNR is Medium \& Delay is HighD \& VelocityDiff. is SmallVD THEN Fuzzy_Cost is Medium R17: IF SNR is Medium \& Delay is HighD \& VelocityDiff. is MediumVD THEN Fuzzy_Cost is Low R18: IF SNR is Medium \& Delay is HighD \& VelocityDiff. is LargeVD THEN Fuzzy_Cost is Medium R19: IF SNR is High \& Delay is LowD \& VelocityDiff. is SmallVD THEN Fuzzy_Cost is Medium R20: IF SNR is High \& Delay is LowD \& VelocityDiff. is MediumVD THEN Fuzzy_Cost is High R21: IF SNR is High \& Delay is LowD \& VelocityDiff. is LargeVD THEN Fuzzy_Cost is VHigh R22: IF SNR is High \& Delay is MediumD \& VelocityDiff. is SmallVD THEN Fuzzy_Cost is Medium R23: IF SNR is High \& Delay is MediumD \& VelocityDiff. is MediumVD THEN Fuzzy_Cost is High R24: IF SNR is High \& Delay is MediumD \& VelocityDiff. is LargeVD THEN Fuzzy_Cost is VHigh R25: IF SNR is High \& Delay is HighD \& VelocityDiff. is SmallVD THEN Fuzzy_Cost is Low R26: IF SNR is High \& Delay is HighD \& VelocityDiff. is MediumVD THEN Fuzzy_Cost is Medium R27: IF SNR is High \& Delay is HighD \& VelocityDiff. is LargeVD THEN Fuzzy_Cost is High

Figure 11. Knowledge structure based on fuzzy rules

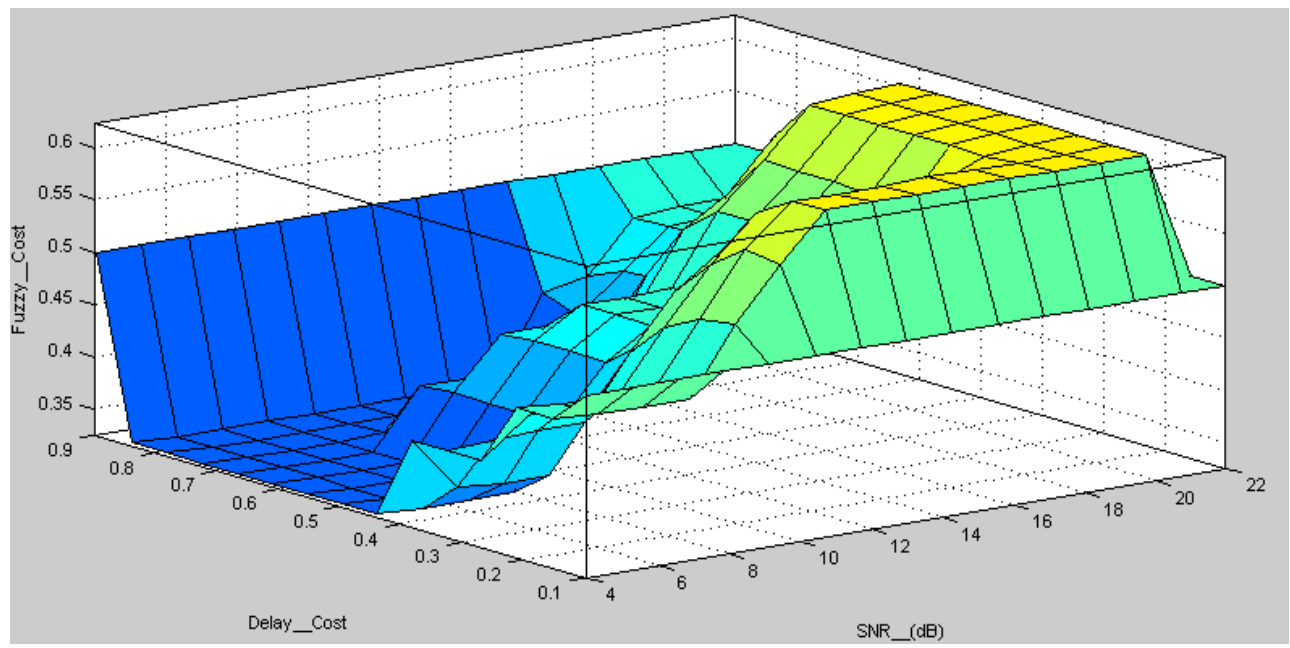

Figure 12. Correlation between input (SNR and Delay_Cost) and output fuzzy variables. 


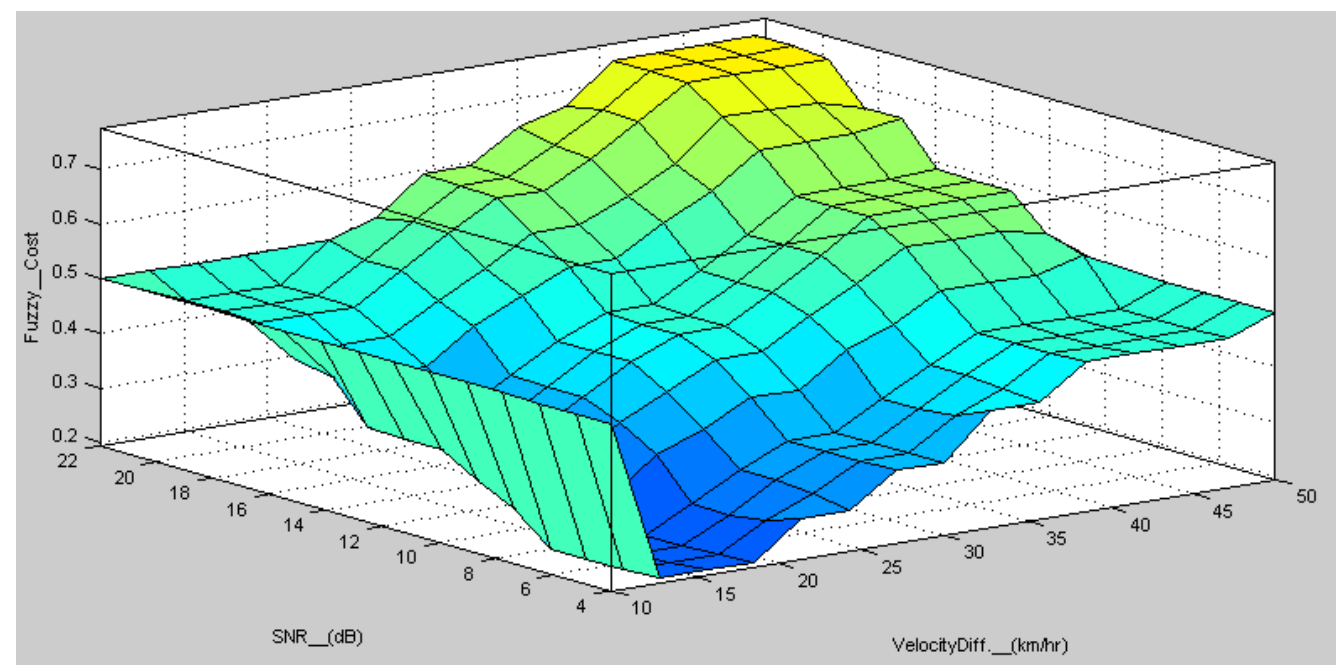

Figure 13. Correlation between input (SNR and VelocityDiff.) and output fuzzy variables.

This value (0.546) represents the FuzzyCost of the specified path. The FuzzyCost which has this value is due to the high value of path Delay. It means our fuzzy inference system uses a compromised decision based on routing metrics (SNR,Delay and Velocity Vector) to select the best path. This output is achieved by using Mamdani's fuzzy inference method [25]. The Fig. 12 and Fig. 13 depicts the correlation behavior between input and output variables. In Fig.12 the trend shows that the value of output FuzzyCost decreases as the SNR decreases as well as path delay between neighbor vehicles increases. This is because the higher path delay or lower SNR leads to lower probablity of fuzzy path selection (dark blue part). In Fig.13 as both velocity difference and SNR increases the probablity of fuzzy decision increases (dark yellow part)

\subsubsection{Defuzzification}

Defuzzification refers to the way a crisp value is extracted from a fuzzy set as a representation value. In our fuzzy decision making, we take the centroid of area strategy for defuzzification. This defuzzifier method is based on (1), as under

$$
\text { Fuzzy_Cost }=\left[\sum_{\text {All Rules }} x i * \mu(x i)\right] /\left[\sum_{\text {All Rules }} \mu(x i)\right.
$$

where Fuzzy_Cost is used to specify the degree of decision making, xi is the fuzzy variable and $\mu(\mathrm{xi})$ is its membership function. Based on this defuzzificatioon method, the output of the fuzzy cost function is changed to the crisp value.

In addition, the performance of fuzzy logic decision making system depends upon determining the fuzzy set of membership functions. This fuzzy set is often built by designers with trial and error or based on their experiences. However, VANET poses rapid changing of topology and rapid arrival or departures. For this reason, automatic tuning (refining the fuzzy sets) of membership function parameters is necessary. In our proposed protocol, we have 


\section{Macrothink}

used H-Infinity to tune the membership functions according to VANET characteristics.

\subsubsection{Hळ filtering for fuzzy membership function optimization}

$\mathrm{H} \infty$ filtering is an optimization method that takes into consideration a firm mathematical definition of the constraints on the expected behavior of the closed loop control system. To tune the membership functions of fuzzy variables, first we have defined the initial fuzzy set of input/output membership functions (Fig. 9, 10), to make the tuning procedure easier. We then use $\mathrm{H} \infty$ filtering as a linear estimator; it tunes the membership functions according to the vehicular scenario. We used this technique because of its robustness to system noise, modeling errors and nonlinearities [26]. This technique is used periodically to tune the membership functions of the fuzzy inference system. This periodic tuning is based on the threshold value of network throughput. We briefly discuss the H-infinity algorithm. Consider a non-linear discrete time system [27] as under (2):

$$
\begin{aligned}
& \mathrm{x}_{i+1}=f\left(\mathrm{x}_{i}\right)+B \omega_{i}+\rho_{i} \\
& \mathrm{y}_{i}=h\left(\mathrm{x}_{i}\right)+\mathrm{v}_{i}
\end{aligned}
$$

Where, the $x_{i}$ is the state of the system at time $i, \omega_{i}$ is the process noise, $y_{i}$ is the observation vector, $v_{i}$ is the observation noise, $\rho_{i}$ is an arbitrary noise sequence and $f\left(x_{i}\right)$ and $\mathrm{h}\left(\mathrm{x}_{i}\right)$ are non linear vector functions of the state. The problem addressed by the $\mathrm{H} \infty$ is to find an estimate $\bar{x}_{i+1}$ of $x_{i+1}$ given $y_{k}$ where $k=0, \ldots i$ [28]. The augmented noise vector and the estimation error are defined as follow:

$$
\begin{gathered}
\mathrm{e}_{i}=\left(\omega^{T} v^{T}\right)^{T} \\
\tilde{\mathrm{x}}_{i}=\mathrm{x}_{i}-\overline{\mathrm{x}}_{i}
\end{gathered}
$$

The problem solved by the Hळ filter is to find an estimate $\bar{x}_{i}$ such that the infinity norm of the transfer function from the augmented noise vector $\mathrm{e}$ to the estimation error $\mathrm{x}_{\mathrm{i}}$ is bounded by a user-defined quantity $\beta$ :

$$
\|G\| \infty<\beta
$$

Where, the maximum steady state gain from e to $\mathrm{x}$ is less than $\beta$. The desired estimate $\mathrm{x}_{\mathrm{i}}$ can be obtained by the following recursive $\mathrm{H} \infty$ estimator [27]:

$$
\begin{aligned}
& \mathrm{F}_{i}=\left.\frac{\delta f(x)}{\delta x}\right|_{\mathrm{x}=\overline{\mathrm{x}}_{i}} \\
& \mathrm{H}_{\mathrm{i}}^{\mathrm{T}}=\left.\frac{\delta f(x)}{\delta x}\right|_{\mathrm{x}=\overline{\mathrm{x}}_{i}} \\
& \mathrm{Q}_{0}=\mathrm{E}\left(\mathrm{x}_{0} \mathrm{X}_{0}^{\mathrm{T}}\right) \\
& \mathrm{Q}_{\mathrm{i}+1}=\mathrm{F}_{\mathrm{i}} \mathrm{P}_{\mathrm{i}} \mathbf{F}_{\mathrm{i}}^{\mathrm{T}}+\mathrm{B} B^{T} \\
& \mathrm{~K}_{\mathrm{i}}=\mathrm{F}_{\mathrm{i}} \mathrm{P}_{\mathrm{i}} \mathbf{H}_{\mathrm{i}}^{\mathrm{T}} \\
& \overline{\mathrm{x}}_{i+1}=\mathrm{F}_{\mathrm{i}} \overline{\mathrm{x}}_{i}+\mathrm{K}_{\mathrm{i}}\left(\mathrm{y}_{\mathrm{i}}-\mathrm{H}_{\mathrm{i}} \overline{\mathrm{x}}_{i}\right)
\end{aligned}
$$


Assume that the variables $Q_{i}$ and $P_{i}$ are nonsingular sequences of matrices. $K_{i}$ is known as the $\mathrm{H} \infty$ gain.

The optimization of membership functions starts by first initializing the fuzzy set for input/output fuzzy variables as it is demonstrated in Fig. $(9,10)$. All vehicles in the simulated urban scenario trigger their $\mathrm{H} \infty$ technique periodically. The reason of periodic membership tuning is to reduce the time delay of $\mathrm{H} \infty$ technique execution during complete path setup. After triggering the $\mathrm{H} \infty$ technique, the membership functions are parameterized to three parameters; centroid $\left(c_{i j}\right)$, lower half width $\left(a_{i j}\right)$ and upper half width $\left(b_{i j}\right)$ of $i^{\text {th }}$ membership function and $j^{\text {th }}$ input/output variables [27] (Fig.14). Thus, by tuning the values of $c_{i j}, a_{i j}$ and $b_{i j}$ we can optimize the membership functions of fuzzy variables.

\subsection{DR 2 Protocol Operations}

The routing operation starts when a source node has data to transmit to a certain destination and it is out of coverage to that destination. When data packets are sent from a source vehicle, the information like destination location, destination velocity vector, destination ID and message Time To Live (TTL) are written into the packet header (Fig. 15) and then fuzzy inference system selects the best preferable path based on three proposed selection criteria among neighbors. After the best path has been found, the vehicle transmits the information to a selected relay vehicle. The selected relay vehicle repeats the process of selection based on fuzzy inference system. To limit the transmission of the data message in the network, the relay vehicle decrements TTL and transmits the packet to the next relay vehicle or drops the data packets if the result exceeds the data packet life time. The other information like location, velocity and ID of the destination is never changed while the packet is travelling. This behavior does not negatively affect the progress of finding destination while the vehicle's movement is restricted to linear paths in urban moderate mobility.

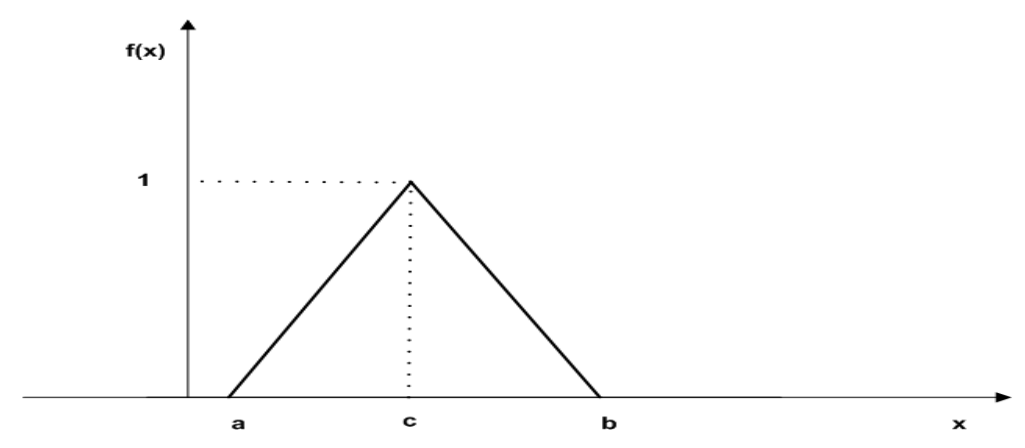

Figure 14. Parameterize of triangular membership functions.

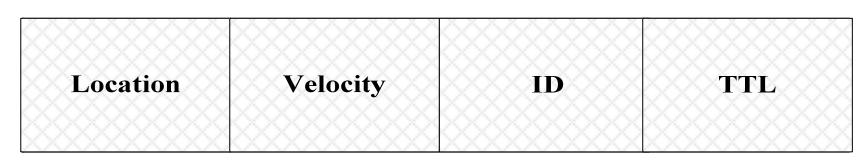

Figure 15. The attached entries of data packet header 
When the relay vehicle/vehicles, in multi-hop fashion, enter the communication range of the destination, the relay vehicle forwards the data packets to the destination without triggering the fuzzy inference technique. The reason of this is to make the relay vehicle transmits data packets to the destination rather than the best preferable path.

\subsection{Performance Evaluation}

In the following sections, through simulation based study, we emphasize the ability of the proposed $\mathrm{DR}^{2}$ protocol in terms of its efficiency and reliability under lossy wireless channel, better end-to-end delay in sparse traffic conditions and high packet delivery ratio. It is worth to mention that simulation based study has been experimented through various traffic densities and degrees of standard deviation.

\subsubsection{Simulation Setup}

In this section, we present the simulation setup to evaluate the performance of the proposed $\mathrm{DR}^{2}$ protocol. The simulation was conducted on (NS-2) [29] with added support IEEE 802.11 DCF as MAC layer. The Simulation of Urban Mobility (SUMO) tool [30] has been employed to create the urban scenario, and to generate mobility traces of the simulated vehicles. SUMO interposes vehicles in each lane (three lane) at a given traffic rate with different speed. This mobility model allows us to simulate vehicular scenarios such as different deceleration/accelerations and various traffic conditions. After the mobility trace file was generated, it was employed by NS to simulate vehicular network scenario.

The parameters related to mobility model and wireless communications are shown in Table 1. We used the simulation area $2400 \times 2400 \mathrm{~m}^{2}$ with maximum travelling speed of 60 $\mathrm{km} / \mathrm{hr}$., the number of vehicles travelling on the roads ranges $50-200$, the data packet size is 1000 bytes and data rate is $2 \mathrm{Mb} / \mathrm{s}[31,32]$. In this scenario all vehicles are communicating with transmission range of 250 meters $(24.50 \mathrm{dBm})$. In order to make the results more precise, each simulation experiment is executed more than one time with each simulation execution lasting 100 seconds. Later on all the results were averaged. 
Table 1. Mobility \& wireless communication related parameters

\begin{tabular}{|l|l|}
\hline Parameter & Settings \\
\hline Simulation area & $2400 \times 2400 \mathrm{~m}^{2}$ \\
\hline Max. Speed limit & $60 \mathrm{~km} / \mathrm{hr}$. \\
\hline Simulation time & $100 \mathrm{~s}$ \\
\hline Propagation model & Shadow fading \\
\hline Traffic density & $50 \sim 200$ \\
\hline Transmission range & 250 meter \\
\hline Transmitting power & $24.50 \mathrm{dBm}$ \\
\hline data rate & $2 \mathrm{Mb} / \mathrm{s}$ \\
\hline Packet size & $1000 \mathrm{byte}$ \\
\hline Beaconing frequency & $0.5 \mathrm{~s}$ \\
\hline Traffic model & $\mathrm{CBR} / 10$ random source \\
\hline Number of lanes & $1 * 3$ \\
\hline $\begin{array}{l}\text { Triggering frequency } \\
\text { for tuning }\end{array}$ & $1 \mathrm{~s}$ \\
\hline
\end{tabular}

In order to model the channel as lossy channel we use log-normal shadow fading. Shadowing effect states that at any given distance $d$ is the distance between transmitter and receiver from a transmitter, the path loss PL(d) at particular location is random and distributed log-normally about the mean distance-dependent value [31]. Therefore, path loss at $d$ meter from the transmitter is modeled as follows:

$$
\mathrm{PL}(d)[d B]=P L\left(\mathrm{~d}_{0}\right)+10 n \log \left(\frac{d}{\mathrm{~d}_{0}}\right)+\mathrm{X}_{\sigma}
$$

Where $\operatorname{PL}\left(\mathrm{d}_{0}\right)$ the average path loss at a reference distance is $\left(\mathrm{d}_{0}\right), \mathrm{n}$ is the path loss exponent which indicates the fluctuation of path loss with distance, $\mathrm{X}_{\sigma}$ is a zero mean Gaussian distributed random variable with standard deviation $\sigma$. We set $n=3$ for path loss exponent and $\mathrm{d}_{0}$ is 0.4 for reference distance. To evaluate the proposed protocol with different channel conditions, we vary the shadow standard deviation $\sigma$.

\subsubsection{Simulation Results}

The following metrics are considered in our evaluation: Packet Delivery Ratio (PDR): measures the fraction of data packets that are successfully delivered to the destination; End to End delay: this value measures the total delay time from a sender to a destination. We compared our results with AODV [15] and GPSR [19] protocols, to investigate the effect of network metrics on the routing protocols.

By changing the degree of standard deviation $\sigma$ we can adjust the error of wireless channel. Fig. 16 shows the packet delivery ratio for urban scenario with different standard deviation $\sigma$ with a fixed number of 100 vehicles. This figure demonstrates the effectiveness of our fuzzy based path selection (SNR is one of the path metrics). As the channel error 


\section{Macrothink}

increases, the average PDR of both AODV (39\%) and GPSR (21\%) decreases. However, DR ${ }^{2}$ (75\%) perform better at all degrees of channel error.

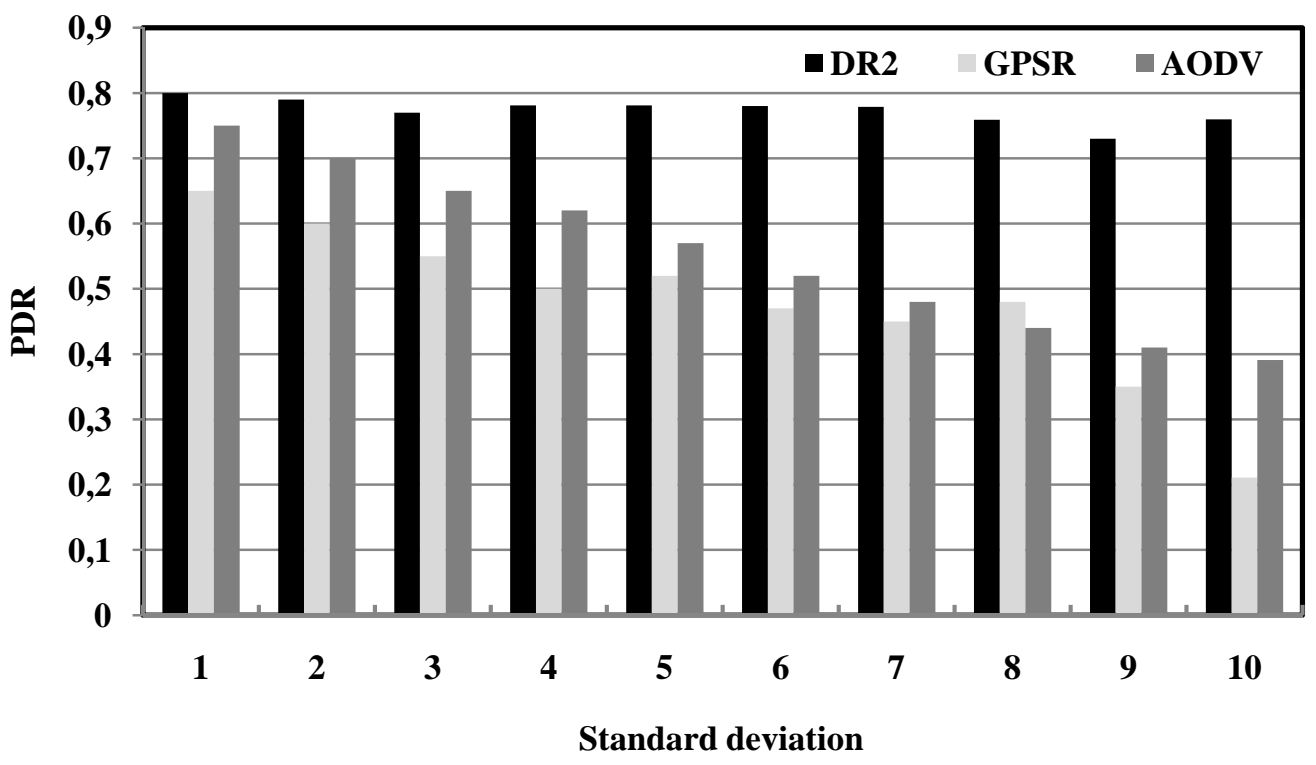

Figure 16. Packet delivery ratio with different channel error conditions (100 vehicles)

The reason for this difference is that our $\mathrm{DR}^{2}$ path selection is based on cross layer design in which MAC layer collects path metrics and network layer selects the best preferable path based on fuzzy system (SNR, delay and velocity difference are the parameters). It is a well known fact, higher SNR, lower delay and may higher velocity difference leads to higher reception quality. This is because the relay vehicle with large velocity difference may it is farthest or nearest node from the source. On the other hand, GPSR performed poorly in comparison to AODV, this is because it chooses from a group of neighbor vehicles the farthest possible hop, which leads to higher attenuation, and hence higher packet loss.

We observed similar trends in PDR as we increased number of vehicles to 200 with varied degree of standard deviation (Fig. 17). When the network becomes more and more connected, the opportunity for packets to be forwarded to the destination increases. As a result, the number of successful delivered data packets increases too.

Another interesting metric is the end-to-end delay of the data packets. The result of this metric is shown in Fig. 18; as the traffic condition becomes more and more connected (dense), the $\mathrm{DR}^{2}$, GPSR and AODV have more neighbors to maintain. Thus, the bandwidth overhead also increases. Similarly, the end to end delay also increases with increasing number of vehicles. In $\mathrm{DR}^{2}$, as the number of vehicles increases, the time required to collect routing metrics (SNR, delay and velocity difference) also increases. The determination of these metrics of the neighbor vehicles becomes more time consuming due to reactively calculating the routing metrics by relay vehicles. In addition to that, the general upward trend of end-to -end delay is due to the fact that there is now a connected path which drives the average delay up. As a result, end to end delay of $\mathrm{DR}^{2}$ increases (see Figure 18). Like $\mathrm{DR}^{2}$, GPSR consumes extra time to determine which vehicle in neighborhood is closer to destination. For 
AODV, the route discovery and reply process adds delay to the data packets. The $\mathrm{DR}^{2}$ protocol determines three metrics for path selection while GPSR tries to find the neighbor node which should be closer to packet destination. Therefore, in dense traffic condition our $\mathrm{DR}^{2}$ protocol performed poorly in comparison of GPSR and AODV. However, in sparse traffic condition our protocol maintains the end to end delay lower that GPRS and AODV.

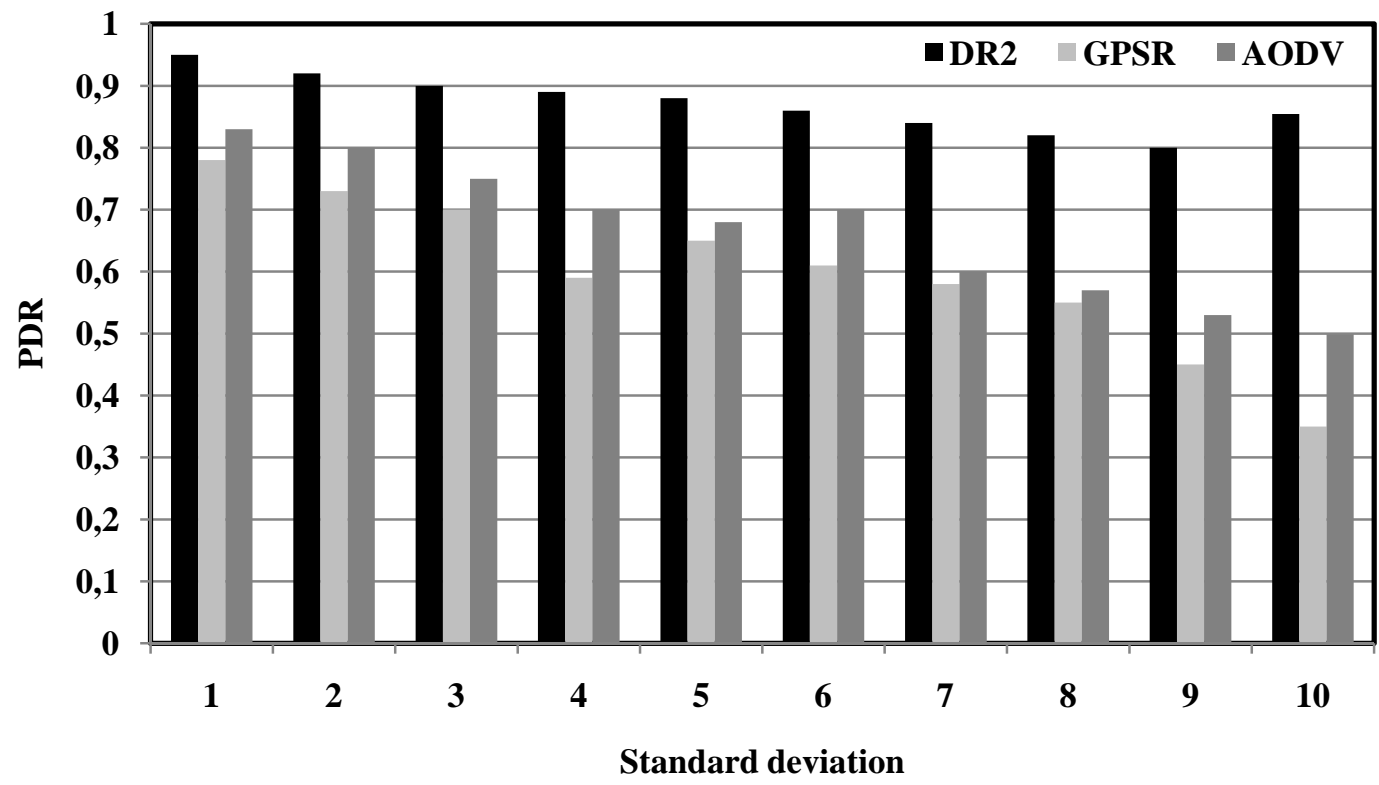

Figure 17. Packet delivery ratio with different channel error conditions (200 vehicles)

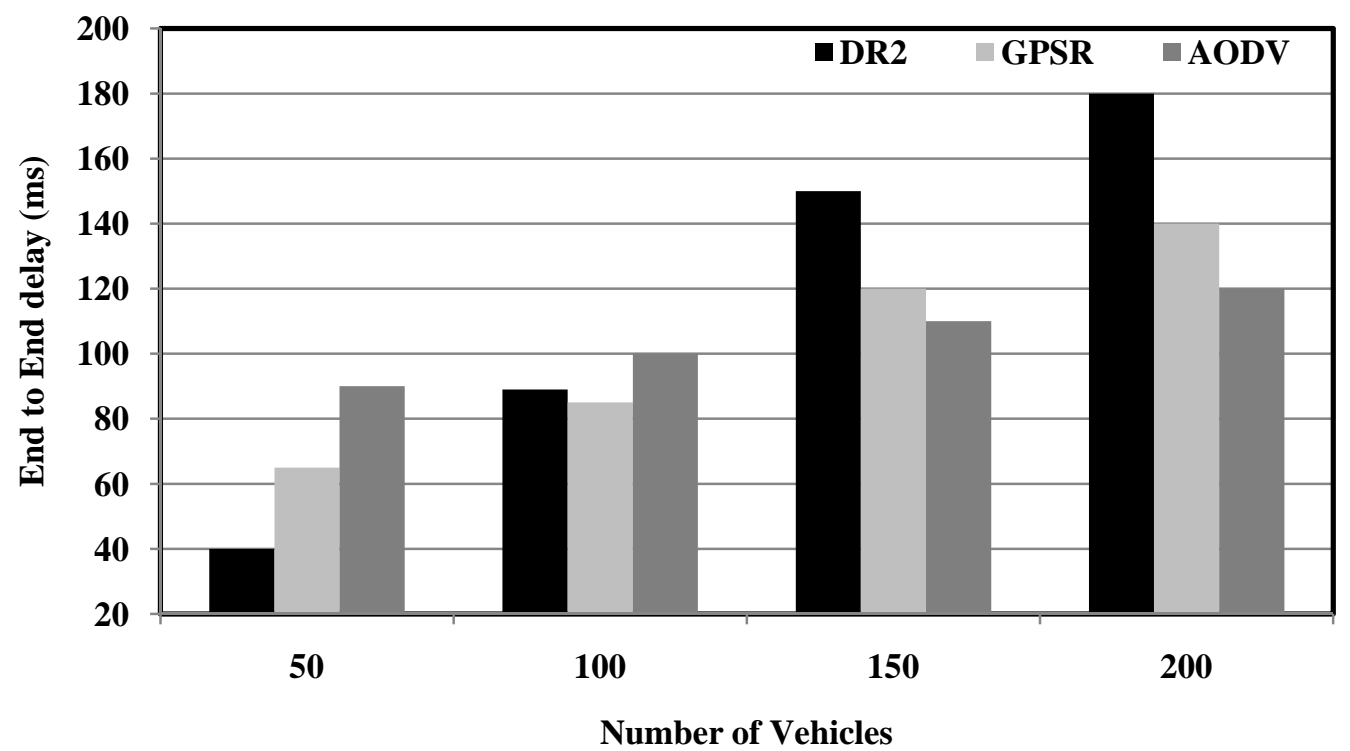

Figure 18. End-to end delay with different vehicular traffic densities

\section{Conclusions}

In this study a review about routing protocols and algorithm has been done for video transmission and text routing over VANETs. Considering the guide lines of the review, we have proposed Delay and Reliability aware Routing $\left(\mathrm{DR}^{2}\right)$ protocol that routes data packets 
over the best preferable path. The proposed protocol has been designed successfully based on cross layer communication between the MAC and the network layers that uses three routing metrics of neighboring vehicles to select the best preferable path. We also successfully used $\mathrm{H} \infty$ technique to tune and optimize the membership functions of the designed fuzzy inference system. Also, NS-2 simulations were successfully carried out for significant gains of $\mathrm{DR}^{2}$ in terms of reliability under erasure channel and low delay in sparse traffic conditions. Thus our approach can be used in real time systems under lossy wireless channel and sparse vehicular traffic conditions.

\section{Acknowledgement}

The authors would like to thank to the administration of Faculty of Computer Science \& Information Systems and Universti Teknologi Malaysia for their generous support.

\section{References}

[1] "Car-to-Car communication," [Online]. Available: http://www.car-to-car.org. [Accessed: 15 June, 2010].

[2] "US department of transportation: Intelligent transportation systems," [Online]. Available: http://www.its.dot.gov.[Accessed: 10 June,2010].

[3] "Ahsra: Advance cruise-assist highway system research association," [Online]. Available: http://www.ahsra.or.jp.[Accessed : 15 June,2010].

[4] Fan, L. and YU, W., "Routing in vehicular ad hoc networks: a survey," IEEE Vehicular Technology Magazine, pp. 12-22, 2007.

[5] Korkmaz G. et al., "Urban Multi-hop Broadcast protocol for inter-vehicle Communication systems, " in 1st ACM International Workshop on Vehicular Ad Hoc Networks, Philadelphia, PA, USA, pp. 76-85,2004.

[6] Yang, X. et al., "A Vehicle-to-Vehicle communication protocol for cooperative collision warning," The First Annu. Int. Conf. Mobile Ubiquitous Syst.: Netw. Services, pp. 114-123. 2004.

[7] Fasolo, E., Furiato, R. and Zanella, A., "Smart Broadcast for inter-vehicular communications," The Proc. of WPMC05, Alborg Denmark, Sep.2005.

[8] Zhang, X., Xie, F., Wang, W. and Chatterjee, M., "TCP throughput for Vehicle-to-Vehicle communications," The First International Conference on Communications and Networking in China (China COM), pp. 1-5. Oct. 2006.

[9] Chen, Z., Kung H. and Vlah, D., "Ad hoc relay wireless networks over moving vehicles on highways," The International Conference of Mobihoc, pp. 247-250, 2001.

[10] Little, T.D.C. and Agarwal, A., "An information propagation scheme for VANETs," The 8th International IEEE Conference on Intelligent Transportation Systems (ITSC 2005), pp. 155-160, 2005.

[11] Francisco, R. J., Pedro, R. M., and Ivan S. , "Reliable and efficient broadcasting in Vehicular Ad Hoc Networks" The 69th IEEE Conference on Vehicular Technology, 
Barcelona, Spain, 2009.

[12] Peiyuan, L., Xinhong, W., Ning, XL., and Fuqiang, L., "A reliable broadcast routing scheme based on mobility prediction for VANET" The International Conference on Intillegent Vehicles, p.p 1083-1087, 2009.

[13] $\mathrm{Wu}, \mathrm{H}$. et al., "MDDV: a mobility-centric data dissemination algorithm for vehicular Networks," The First ACM International Workshop on Vehicular Ad Hoc Networks (VANET'04), Philadelphia, PA, USA, pp. 47-56,2004.

[14] Maihöfer, C., "A survey of geocast routing protocols," IEEE Commun. Surveys Tutorials, vol. 6, pp. 32-42, 2004.

[15] Perkins, C. E. and Royer, E. M. "Ad Hoc On-Demand Distance Vector Routing," The IEEE International Workshop WMCSA, New Orleans, LA, PP. 10-100, 1999.

[16] Maihöfer, C., Leinmüller, T. and Schoch, E., "Abiding geocast: time-stable geocast for ad hoc networks," The 2nd ACM international workshop on Vehicular ad hoc networks (VANET '05), pp. 20-29, 2005.

[17] Qiangyuan, Y., Geert, H., "Abiding geocast for warning message dissemination in vehicular ad hoc networks," The IEEE International Conference on Communications, Beijing, China, pp. 400-404, 2008.

[18] Taleb, T., Ochi, M., Kato, N., and Nemoto, Y., "An efficient vehicle-heading based routing protocol for VANET networks," The IEEE Wireless Communications and Networking Conference, WCNC 2006.

[19] Karp, B. and Kung, H.T. "GPSR: Greedy perimeter stateless routing for wireless networks," The ACM/IEEE International Conference on Mobile Computing and Networking (MobiCom), 2000.

[20] Lochert, C., Mauve, M., Füßler, H. and Hartenstein, H., "Geographic routing in city scenarios," ACM SIGMOBILE Mobile Computing and Communications Review (MC2R), vol. 9, no. 1, pp. 69-72, January 2005.

[21] Lee, K. C., Haerri, J., Lee, U and Gerla, M., "Enhanced perimeter routing for geographic routing protocols in urban vehicular scenarios," The IEEE Autonet workshop, 2007.

[22] Zhao, J. and Cao, G., "VADD: Vehicle-assisted data delivery in vehicular ad hoc networks," The IEEE Infocom, 2006.

[23] Guha, K. R. and Chen, W. "Achieving high-rate multi-hop data delivery in vehicular networks," The 69th IEEE Conference on Vehicular Technology, 2009.

[24] Adya, A., et al.,"A multi-radio unification protocol for IEEE wireless networks" The First International Confereence on Broadband Networks, Washington, pp. 344-354,2004.

[25] E.H. Mamdani,"Application of fuzzy logic to approximate reasoning using linguistic synthesis," IEEE Trans. on Computers, vol. C-26, pp.1182-1191, Dec. 1977.

[26] D. Simon and H. El-Sherief, Hybrid Kalman/Minimax Filtering in Phase-Locked Loops, Control Engineering Practice,vol. 4, no. 5, 615-623, 1996.

[27] Zhou,S. and Feng, G., "H-infinity filtering for discrete-time systems with randomly varying sensor delays" Automatica, pp.1918-1922, 2008.

[28] Kharrati, H., and Khanmohammadi, S., " Genetic algorithm combined with $\mathrm{H} \infty$ filtering for optimizing fuzzy rules and membership functions”, Journal of Applied Science, 


\section{Macrothink}

Network Protocols and Algorithms

ISSN 1943-3581

2010, Vol. 2, No. 2

pp.3439-3445,2008.

[29] NS simulator, http://nsnam.isi.edu/nsnam/index.php/Main_Page, accessed: 25 May 2010.

[30] Simulation of Urban Mobility (SUMO), http://sumo.sourceforge.net, accessed: 25 May 2010 .

[31]Jarupan, B. and Ekici, E., "Location- and delay-aware cross-layer communication in V2I multihop vehicular networks", IEEE Communication Magazine, pp. 112-118, November 2009.

[32] Gong, J., et al., "Predictive directional greedy routing in vehicular ad hoc networks", The IEEE International Conference on Distributed Computing Systems Workshops, 2007.

[33] Rappaport, S. Theodore, "Wireless communication principles and practice", $2^{\text {nd }}$ Edition, Pearson Education Inc., pp. 138-144,2002. 